\title{
Intact glucose uptake despite deteriorating signaling in adipocytes with high-fat feeding
}

\author{
Björn Hansson', Sebastian Wasserstrom', Björn Morén', Vipul Periwal², Petter Vikman³, Samuel W Cushman², \\ Olga Göransson', Petter Storm ${ }^{3}$ and Karin G Stenkula'
}

1Department of Experimental Medical Science, Lund University, Lund, Sweden

${ }^{2}$ National Institute of Diabetes and Digestive and Kidney Diseases, National Institutes of Health, Bethesda, Maryland, USA

${ }^{3}$ Department of Clinical Sciences, Lund University, Malmö, Sweden

Correspondence should be addressed to K G Stenkula: karin.stenkula@med.lu.se

(P Storm is now at Department of Experimental Medical Science, Lund University, Lund, Sweden)

\begin{abstract}
To capture immediate cellular changes during diet-induced expansion of adipocyte cell volume and number, we characterized mature adipocytes during a short-term high-fat diet (HFD) intervention. Male C57BL6/J mice were fed chow diet, and then switched to HFD for 2, 4, 6 or 14 days. Systemic glucose clearance was assessed by glucose tolerance test. Adipose tissue was dissected for RNA-seq and cell size distribution analysis using coulter counting. Insulin response in isolated adipocytes was monitored by glucose uptake assay and Western blotting, and confocal microscopy was used to assess autophagic activity. Switching to HFD was accompanied by an immediate adipocyte size expansion and onset of systemic insulin resistance already after two days, followed by recruitment of new adipocytes. Despite an initially increased non-stimulated and preserved insulin-stimulated glucose uptake, we observed a decreased phosphorylation of insulin receptor substrate-1 (IRS-1) and protein kinase B (PKB). After 14 days of HFD, both the insulin-stimulated phosphorylation of Akt substrate of $160 \mathrm{kDa}$ (AS160) and glucose uptake was blunted. RNA-seq analysis of adipose tissue revealed transient changes in gene expression at day four, including highly significant upregulation of Trp53inp, previously demonstrated to be involved in autophagy. We confirmed increased autophagy, measured as an increased density of LC3-positive puncta and decreased p62 expression after 14 days of HFD. In conclusion, HFD rapidly induced systemic insulin resistance, whereas insulin-stimulated glucose uptake remained intact throughout 6 days of HFD feeding. We also identified autophagy as an early cellular process that potentially influences adipocyte function upon switching to HFD.
\end{abstract}

Journal of Molecular Endocrinology (2018) 60, 199-211

\section{Introduction}

It is well known that dysfunctional adipose tissue profoundly influences whole-body metabolism, and obesity is one of the main risk factors related to insulin resistance and type 2 diabetes. Hence, further exploration of adipocyte function is urgently needed.

\section{Key Words \\ - adipocytes \\ - insulin signaling \\ - autophagy \\ - glucose uptake}


1983, Yang et al. 2012) and to be less responsive to insulin (Salans et al. 1968, Salans \& Dougherty 1971, Smith 1972, Franck et al. 2007). Hypertrophic cells have also been shown to trigger an inflammatory response within the adipose tissue, which in turn impairs the insulin signaling in mature adipocytes (Rotter et al. 2003). Adipocyte size, rather than the degree of obesity, has been shown to predict type 2 diabetes (Weyer et al. 2000), and impaired recruitment of new, small adipocytes correlated with insulin resistance in equally obese subjects (McLaughlin et al. 2007). Independent of the cell size, numerous studies have reported impaired insulin signaling at different levels in adipocytes from obese and insulin-resistant subjects (Shao et al. 2000, Danielsson et al. 2005, 2009). Nevertheless, the cellular events preceding the onset of obesity-induced insulin resistance in adipocytes are not yet resolved.

High-fat diet (HFD) feeding of mice is a robust model commonly used to study insulin resistance and obesity (Winzell \& Ahren 2004). Recently, we demonstrated a delay of three days before recruitment of new adipocytes when switching to a HFD (Li et al. 2016). In another study, short-term (15 days) overfeeding was associated with increased autophagy (Nunez et al. 2013), a process tightly linked to organelle recycling and lipid metabolism. These data are in line with studies in humans, where increased autophagic flux was observed in adipocytes from obese and type 2 diabetic subjects (Ost et al. 2010, Kovsan et al. 2011). Kovsan and coworkers demonstrated that increased autophagy correlated with both the degree of obesity and adipose cell hypertrophy (Kovsan et al. 2011). In contrast, autophagic flux has also been shown to be reduced in adipocytes from obese subjects (Soussi et al. 2015), and there is no conclusive evidence as to whether increased autophagy is beneficial or detrimental to adipocyte function.

Even though HFD feeding is commonly used for exploring obesity-associated mechanisms, few studies have addressed the initial changes of cellular metabolism in isolated cells following over-feeding. In order to capture the early cellular changes during diet-induced expansion of adipose cell volume and number, we have characterized the changes in mature adipocyte metabolism during a 14-day HFD intervention in mice, the time-frame chosen based on our previous knowledge of adipocyte cell expansion and recruitment (Li et al. 2016). We demonstrate that HFD feeding rapidly induced gene expression changes in adipose tissue and that insulininduced phosphorylation of the signaling intermediates IRS-1, PKB and AS160 gradually decreased, whereas insulin-stimulated glucose uptake in adipocytes remained intact during the first six days of HFD. We also observed an increased autophagic activity that coincided with diminished insulin signal transduction.

\section{Materials and methods}

\section{Reagents and chemicals}

Heat shock protein (HSP) 90 antibody was from Sigma, AS160 antibody was from EMD Millipore, purified GLUT4 antiserum from Hoffmann-La Roche (Al-Hasani et al. 2002), GLUT1 antibody was from Santa Cruz, p62, S6K1, S6K1 Thr389, AS160 Thr642, PKB, PKB Ser473, PKB Thr308, and LC3 antibodies raised against LC3A/B were from Cell Signaling Technologies. Proteasomal inhibitor MG132 was from Sigma Aldrich, fluorescence-conjugated secondary antibodies Alexa Fluor-568 and BODIPY from Molecular Probe, bovine serum albumin (BSA) from Celliance (Toronto, Canada), NEC042 and NEC3770 were from Perkin Elmer, and rapamycin and chloroquine were from Enzo Life Sciences (Farmingdale, NY, USA).

\section{Animals and high-fat diet intervention}

Male C57BL/6J mice (Taconic, Ry, Denmark) were used at 9 weeks of age. Animals were on a 12-h light cycle with non-restricted food and water. Groups of animals ( $n=6-9$ animals/group) were fed chow diet and switched to HFD (D12492 60 E\% fat content; Research Diets, New Brunswick, NJ, USA) as indicated in Fig. 1 (2, 4, 6 or 14 days of HFD, control group fed chow for 14 days). Thus, the feeding protocol and the following experimental

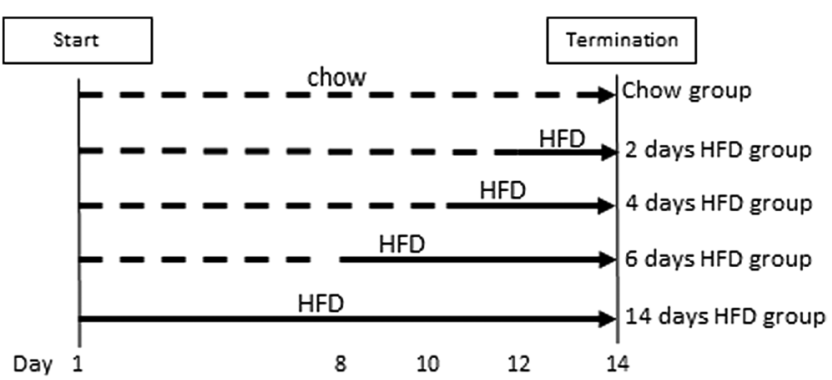

All animals same age Day 1 (male, 9 weeks old, C57BL/6J)

\section{Figure 1}

Study design. Groups of animals (C57/B/6J, male, $n=6-9$ animals/group) were fed chow diet and switched to HFD for 2, 4, 6 or 14 days, a control group was fed chow for 14 days. The feeding protocol was terminated the same day for all groups, and the following experimental analyses conducted included all groups. The protocol was repeated three times, generating three independent experiments. 
assays were terminated at the same day (Fig. 1). The entire feeding protocol was repeated three times, generating three independent experiments. All animal procedures were approved by the Malmö/Lund Committee for Animal Experiment Ethics, Lund, Sweden.

\section{Glucose tolerance test and serum analysis}

Mice fasted overnight (12 h) were injected intraperitoneally (i.p.) with glucose (50 mg/mouse) followed by collection of serum samples at the indicated times. Blood glucose levels were measured (OnetouchUltra2; Lifescan, Milpitas, CA, USA), and insulin levels were assayed in serum using ELISA (Mercodia, Uppsala, Sweden). Terminal serum samples collected were analyzed for TNFalfa and IFNgamma using MSD Multi-spot assay system Proinflammatory panel 1 (mouse) kit, non-esterified free fatty acid (NEFA) kit (Wako) and adiponectin kit (R\&D Systems). All samples were analyzed in duplicate.

\section{Cell size distribution}

Adipose tissue samples were obtained from epididymal fat tissue. The adipose cell-size distributions were obtained using a Beckman-Coulter counter after osmium fixation as described previously (Li et al. 2016).

\section{Isolation of adipocytes}

Primary mouse adipocytes were isolated from epididymal fat tissue as described previously (Rodbell 1964). Isolated cells were suspended $(20 \%(\mathrm{v} / \mathrm{v})$ suspension) in KrebsRinger Bicarbonate HEPES (KRBH) buffer, pH 7.4, containing $200 \mathrm{nM}$ adenosine and 3\% (w/v) BSA.

\section{Glucose uptake and cytosolic volume measurement}

Glucose uptake was determined as previously described (Gliemann et al. 1984), using tracer glucose during a short period of time to minimize the secretion of metabolites. Cells were incubated in $\mathrm{KRBH}$ medium without glucose in triplicate, without or with insulin $(0.01$ or $10 \mathrm{nM})$, for $30 \mathrm{~min}$, followed by addition of ${ }^{14} \mathrm{C}$-D-glucose $(0.5 \mu \mathrm{L} / \mathrm{mL}$, NEC042), and an additional 30-min incubation. The uptake was terminated by spinning $300 \mu \mathrm{L}$ of each cell suspension in microtubes containing $80 \mu \mathrm{L}$ dinonylphtalate oil. The cell fraction was collected, dissolved in scintillation fluid (Optima Gold, Perkin Elmer) and subjected to scintillation counting. Cytosolic volume was assayed using 3-O-methyl glucose uptake (Whitesell \& Gliemann 1979). Cells were incubated in $\mathrm{KRBH}$ medium with methyl-14C-D-glucose (NEC3770) at $37^{\circ} \mathrm{C}$, shaking water bath in triplicate for $30 \mathrm{~min}$. The uptake was terminated as described earlier. Equal volumes of cell suspension were used to measure the lipid contents (Folch et al. 1957).

\section{Western blot}

Adipocytes were incubated with or without insulin as indicated in the figures. To stop incubations, cells were washed in KRBH without BSA, lysed and subjected to polyacrylamide gel electrophoresis and electrotransfer to nitrocellulose membranes as previously described (Lindahl et al. 2015). Briefly, cell lysates (10 $\mu$ g total protein) were heated and subjected to electrophoresis on pre-cast BioRad gradient gels and electrotransfer to nitrocellulose membrane. After blocking and probing with antibodies, detection was performed using horseradish peroxidaseconjugated secondary antibodies and enhanced chemiluminescence reagent, and the signal was visualized and quantified using Biorad camera and image software (Biorad).

\section{RNA seq analysis}

RNA was isolated from epididymal fat tissue ( $n=4$ animals/ per group) using RNeasy Mini Kit (Qiagen) according to the manufacturer's recommendations. Samples were prepared for RNA-seq using Illumina's TruSeq RNA Sample Preparation Kit and sequenced on Illumina HiSeq 2000 with six samples per lane. Resulting reads $(2 \times 100 \mathrm{bp})$ were aligned to the mouse genome (Ensembl release 80) using STAR (Dobin et al. 2013). Uniquely mapped reads were counted against gene annotations from Gencode (version M6) using featureCounts. Gene expression levels were normalized using TMM, and differential expression was assessed using linear modelling and the limma package ( $\mathrm{R}$ version 3.0). All data are available at GEO using the accession number GSE106714.

\section{Induction of autophagy}

In order to investigate autophagic flux, isolated adipocytes from mice fed either chow or HFD for 14 days were incubated with $500 \mathrm{nM}$ rapamycin (Rap) to induce autophagy, $50 \mu \mathrm{M}$ chloroquine (CQ) to inhibit lysosomal degradation of autophagosomes or left untreated (Ctr) for $4 \mathrm{~h}$ at $37^{\circ} \mathrm{C}$ and $5 \% \mathrm{CO}_{2}$. 


\section{Cell preparation for confocal microscopy}

After autophagy induction, cells were washed $2 \times$ in KRBH buffer without BSA, followed by fixation in $4 \%$ paraformaldehyde (PFA) for $6 \mathrm{~min}$, washed $2 \times$ in PBS, followed by blocking and permeabilization in KRBH buffer with $0.1 \%$ Saponin for $30 \mathrm{~min}$. Cells were incubated with LC3 antibody (1:200) for $1 \mathrm{~h}$, washed $2 \times$ in PBS pH 7.4 with $0.1 \%$ Saponin and re-suspended in the same buffer containing Alexa Fluor-568 goat antirabbit IgG secondary antibody (1:300). After $1 \mathrm{~h}$, cells were washed $2 \times$ in PBS. All steps were performed at room temperature.

\section{Confocal imaging}

Imaging was performed using a Nikon A1 plus confocal microscope with a $60 \times$ Apo DIC oil immersion objective with a NA of 1.40 (Nikon Instruments Inc.) and appropriate filter sets. Images were acquired with NISelements, version: 4.50.02, (Laboratory Imaging) using identical acquisition settings. Threshold of images were based on LC3 fluorescence intensity with a lower cutoff at 900 fluorescence intensity (arbitrary units).

Adipose tissue samples were taken from 2 to 3 separate animals for each time point, fixed for $1 \mathrm{~h}$ in $4 \%$ PFA, washed with PBS and stained with BODIPY for $1 \mathrm{~h}$. A number of z-stacks were acquired for each time point with $20 \times$ objective and a representative slice chosen to visualize cell size in the tissues for each time point on HFD.

\section{Pharmacological proteasomal inhibition}

Isolated adipocytes were incubated for $18 \mathrm{~h}$ with the proteasomal inhibitor MG132 $(10 \mu \mathrm{M})$ or vehicle (DMSO) only. The cells were kept in Dulbecco's Modified Eagle Medium (DMEM), (Life Technologies), supplemented with 200 nM (-)-N6-(2-phenylisopropyl) adenosine (Sigma Aldrich), gentamicin $(0.1 \mathrm{mg} / \mathrm{mL})$, (Sigma Aldrich) and $3.5 \% \mathrm{w} / \mathrm{v} \mathrm{BSA}$ at $37^{\circ} \mathrm{C}$ and $5 \% \mathrm{CO}_{2}$.

\section{Statistical analysis}

Analysis was performed by one-way ANOVA and multiple comparisons or Student's $t$-test when appropriate, using GraphPad Prism 6 (Graphpad Software Inc.) software. Significance was determined according to ${ }^{*} P \leq 0.05$, ${ }^{* *} P \leq 0.01,{ }^{* *} P \leq 0.001$ and ${ }^{* * * *} P \leq 0.0001$.

\section{Results}

\section{Adipose tissue expansion and onset of systemic insulin resistance following HFD feeding}

Animals that were switched to HFD as illustrated in Fig. 1, displayed a rapid increase in body and epididymal fat pad weights (Fig. 2A and B). Analysis of the epididymal adipose cell sizes demonstrated a bimodal cell size distribution, with a fraction of so-called small cells defined by the nadir (McLaughlin et al. 2007), which is the lowest point between the two cell populations (indicated with an arrow in Fig. 2C, left panel), and a fraction of large cells to the right of the nadir. The panels in Fig. $2 \mathrm{C}$ display the cell size distribution curves for chow-fed mice compared to mice fed HFD for 4, 6 and 14 days, respectively. Analysis of the distribution curves confirmed a gradual increase in mean cell size in the fraction of large cells (Fig. 2D), as well as an increase in the proportion of small relative to large cells with increasing time on HFD (Fig. 2E). After 4 days of HFD, the population of small cells increased markedly, interpreted to represent newly recruited adipocytes ( $\mathrm{Li}$ et al. 2016). After 14 days of HFD, the average diameter of the large cell population had almost doubled compared to chow ( 60 and $\sim 107 \mu \mathrm{m}$ in diameter, respectively). It is worth noting that, at the same time, the size distribution within the small cell population had broadened markedly, ranging from 20 to $75 \mu \mathrm{m}$ in diameter in the HFD group, compared to chow, which ranged from 20 to $30 \mu \mathrm{m}$ (Fig. 2C, far right panel). The expansion of the large cell population was visualized in intact adipose tissue by confocal microscopy (Fig. 2F). Note, that the small cell population is almost non-detectable using microscopy.

To examine whether the increased adipose tissue mass induced by HFD was associated with systemic insulin resistance, a subset of animals was subjected to a glucose tolerance test (GTT). Both fasting glucose and insulin levels were markedly elevated already after 2 days of HFD and increased further with increasing days of HFD (Fig. 3A and B). In line with these data, a decreased glucose clearance capacity was observed after 2 days of HFD, which deteriorated progressively with increasing days of HFD feeding (Fig. 3C and D). The impaired glucose clearance was associated with increased insulin levels during the GTT (Fig. 3E), which suggests that the animals had become less insulin sensitive. This was confirmed by calculating the quantitative insulin sensitivity check index (QUICKI (Pacini et al. 2013)) (Fig. 3F). Serum analysis revealed no changes in either NEFA, the pro-inflammatory cytokines TNFalfa and IFNgamma or the anti-inflammatory adipokine adiponectin after 14 days of HFD (Fig. 3G). 

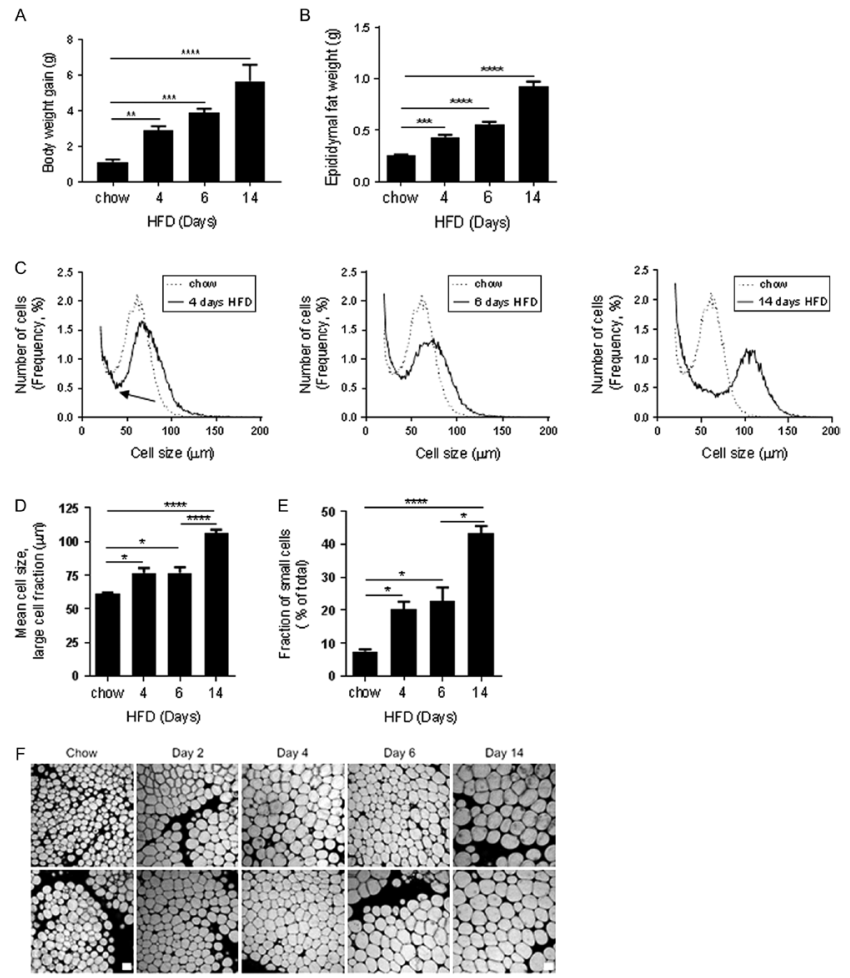

Figure 2

Adipose tissue expansion and onset of systemic insulin resistance following HFD feeding. Body weight gain and epididymal fat pad weights $(\mathrm{g})$ displayed in $(A)$ and $(B)$, respectively. Cell size distribution of fixed intact adipose tissue was measured using a coulter counter, approximately 6000 counts/sample, each sample run in duplicate, $n=4$ samples per feeding group. The average distribution curves, displaying the frequency of cells of varying cell diameter $(\mu \mathrm{m})$, for chow compared with 4, 6 and 14 days of HFD are shown in separate panels. The fraction of so-called small cells to the left of the nadir (arrow), and the fraction of large cells to the right of the nadir (C). Quantification $(n=4)$ of the mean cell size within the large cell fraction, and the proportion of small cells of the total cell population for each feeding condition are shown in (D) and (E), respectively. Representative confocal images (two images per condition), illustrating adipose cell size distribution in intact tissue, stained with BODIPY, scale bar $=50 \mu \mathrm{m}(\mathrm{F})$. Data in (A, B, D and E) presented as mean \pm S.E.M., $* P<0.05, * * * P<0.001, * * * * P<0.0001$.

\section{Intact glucose uptake despite progressively impaired submaximal insulin signaling}

To examine the cellular insulin response, mature adipocytes were isolated from animals fed either chow or HFD for 4, 6 and 14 days and subjected to a tracer-glucose uptake analysis (the 2-days HFD group was excluded from further analyses due to technical limitations). It is important to note that each cell suspension contained mature adipocytes of varying sizes, with a distribution as illustrated in Fig. 2C. In the basal (non-stimulated) condition, the glucose uptake, expressed as uptake/ volume cell suspension, was increased in cells isolated from animals fed HFD for 4 days compared to chow-fed animals (Fig. 4A). In the cell suspension from animals fed a HFD for 6 days, the uptake was decreased compared to the 4-day group, but still significantly increased compared to chow (Fig. 4A), whereas the glucose uptake was similar when comparing chow and the 14-day group. The submaximally and maximally insulin-stimulated uptakes (0.01 nM and 10 nM insulin, respectively) were similar comparing chow, 4 and 6 days of HFD, but there was a decrease in the maximally stimulated uptake in the cell suspension obtained from mice fed HFD for 14 days (Fig. 4A). In parallel, adipocyte cytosolic volume was measured by 3-O-methyl glucose uptake. The cytosolic volume in equal volumes of cell suspension were similar comparing the chow, 4 and 6 days of HFD groups, but significantly decreased after 14 days of HFD compared to chow (Fig. 4B). To explore if the increased glucose uptake in non-stimulated cells following 4 and 6 days of HFD was due to altered expression of glucose transporters, we examined the protein levels of the glucose transporters GLUT1 and GLUT4 in cell lysates prepared for Western blotting from equal cell suspension volumes. There was some variability in the protein levels between the samples within the same feeding group (Fig. 4C), but quantification demonstrated that GLUT1 and GLUT4 proteins were reduced by $\sim 50$ and 30\% respectively after 14 days of HFD compared to chow (Fig. 4D). The total protein content extracted from an equal volume of adipose cell suspension was similar in the different feeding groups (Fig. 4E).

To further explore activation of the insulin signaling pathway leading to glucose uptake, aliquots from the same cell suspensions that were used for glucose uptake analysis were stimulated with insulin $(0,0.001,0.01,0.1$ and $10 \mathrm{nM}$ ) for $20 \mathrm{~min}$, and cell lysates were subjected to Western blotting. Strikingly, the total protein level of IRS-1, the immediate substrate downstream of the insulin receptor, was markedly reduced already after 2 days of HFD, and it decreased even further with increasing days of HFD (Fig. 5A). Insulin-induced phosphorylation of IRS-1 Ser302 was also drastically reduced. In contrast, the total protein expression of PKB and AS160 increased after 14 days of HFD (Fig. 5A). The sub-maximally insulinstimulated phosphorylation of PKB Thr308/Ser473 and AS160 Thr642 gradually decreased with increasing days of HFD. The maximally stimulated phosphorylation of PKB Thr308/Ser473 also decreased, whereas phosphorylation of AS160 Thr642 remained intact at the highest dose of insulin (Fig. 5A). Quantification of data in Fig. 5A confirmed that there was a downward shift in the insulin response with HFD (Fig. 5B). For all HFD groups, there was a marked reduction in PKB phosphorylation (Fig. 5B, 

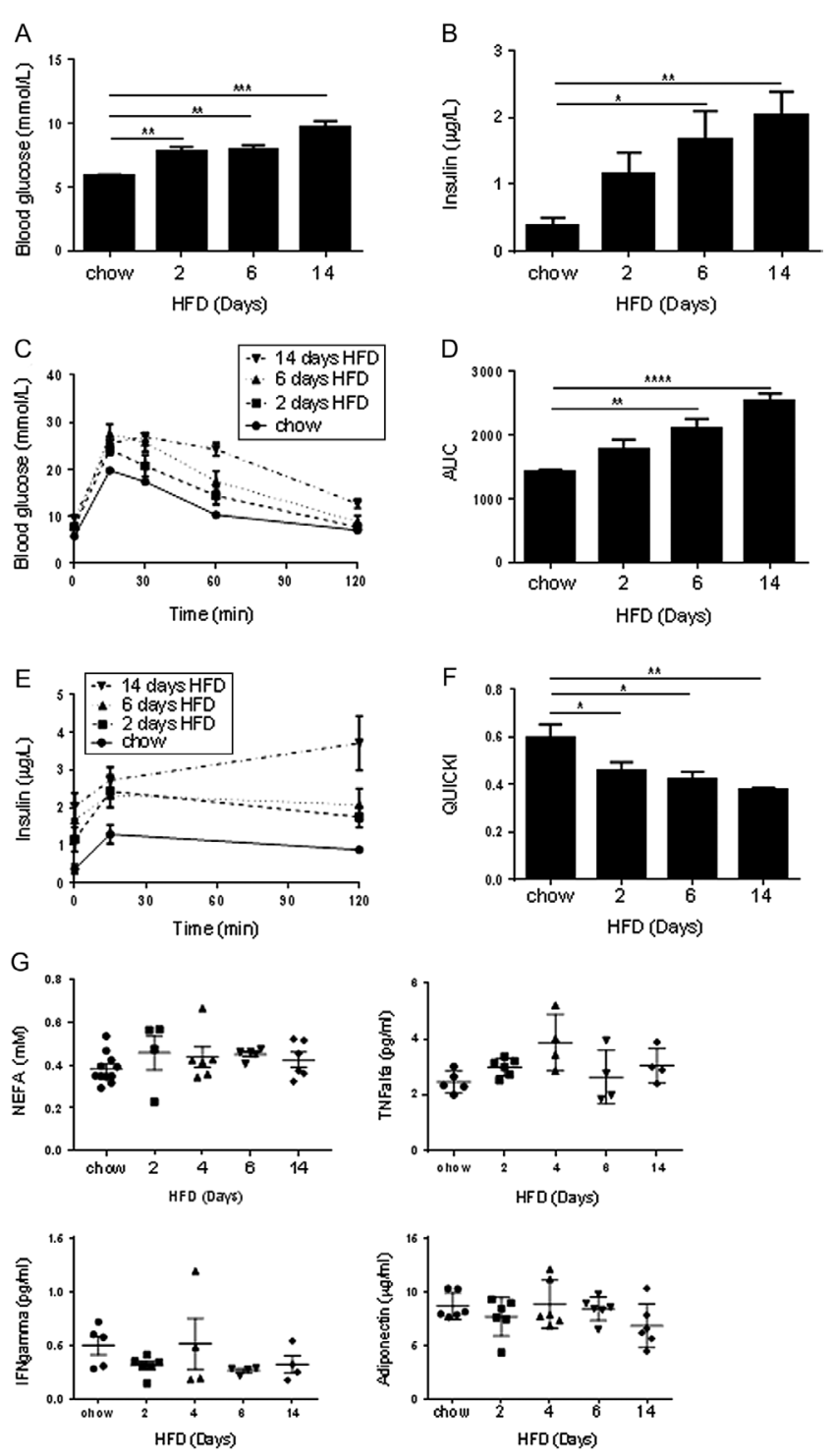

Figure 3

Onset of systemic insulin resistance following HFD. Blood glucose ( $\mathrm{mmol} / \mathrm{L}$ ) and serum insulin ( $\mu \mathrm{g} / \mathrm{L}$ ) in mice ( $n=6$ animals/group) fed HFD for 2, 6 or 14 days, or chow (14 days) displayed in (A) and (B), respectively. Blood glucose ( $\mathrm{mmol} / \mathrm{L}$ ) was measured in serum collected at time point 0 , $15,30,60$ and $120 \mathrm{~min}$ during i.p. glucose tolerance test (GTT), $n=6$ animals/group (C). Data in (C) plotted as AUC (D). Serum insulin levels $(\mu \mathrm{g} / \mathrm{L})$ measured during GTT (E). Quicki calculated based on fasting insulin and glucose levels according to Pacini et al. (Pacini et al. 2013) (F). Serum analysis of IFNgamma, NEFA, TNFalfa, and Adiponectin, $n=4-6$ animals/ group (G). Data in (A, B, C, D, E and F) presented as mean \pm s.E.M., $* P<0.05, * * P<0.01, * * * P<0.001, * * * * P<0.0001$.

left panel). The 4 days of HFD and chow groups displayed similar phosphorylation, both non-stimulated and at the lowest insulin concentrations. For AS160, the 14-day group displayed a markedly reduced phosphorylation in response to insulin, especially at lower insulin concentrations, whereas the other groups displayed similar degree of phosphorylation (Fig. 5B, right panel).

Insulin has previously been shown to cause increased degradation of IRS-1 protein (Buren et al. 2003), and the rapidly diminished expression of IRS-1 protein in adipocytes from HFD-fed mice could be related to the elevated levels of circulating insulin (Fig. 3B). To test this hypothesis, adipocytes from chow-fed mice were treated with $100 \mathrm{nM}$ insulin for $18 \mathrm{~h}$, with or without the proteasome inhibitor MG132. After treatment, cells were lysed and subjected to Western blot analysis. Compared to control cells, there was a marked reduction in the levels of IRS-1 protein in the insulin-treated cells (Fig. 5C). In the presence of MG132 (Fig. 5C), there was no reduction in the levels of IRS-1 in insulin-treated cells compared to controls, supporting the notion that prolonged insulin exposure reduces IRS1-levels by ubiquitination and proteasomal degradation.

\section{Profound transcriptional changes linked to autophagy following HFD feeding}

To capture the early changes in adipose tissue during HFD feeding, we conducted an RNA-seq analysis of intact adipose tissue after 2, 4, 6 and 14 days of HFD compared to control chow feeding for 14 days (as illustrated in Fig. 1). RNA was extracted from the epididymal fat pads of four animals at each time point and sequenced using Illumina technology with an average read depth of $22 \mathrm{M}$ reads per sample. To identify differentially expressed genes, an ANOVA model was fitted to the data and each time point was compared to the control chow-fed animals, designating genes with a false discovery rate (FDR) $q<0.05$ as differentially expressed (DE). 3831 genes were identified as differentially expressed at all time points, with the fifty most significant visualized in a heatmap (Fig. 6A). However, the most dramatic change in gene expression was identified as a marked but transient response at day 4 , with more than 3000 genes identified as DE (Fig. 6B, top 10 listed in Fig. 6C) (full list in Supplementary Table 1 , see section on supplementary data given at the end of this article). To further characterize the transcriptional response to HFD, we subjected the differentially expressed genes at day 4 to transcription factor analysis (Supplementary Fig. 1, TFM Explorer) and gene ontology (GO) enrichment analysis (Supplementary Fig. 2, GO stats). Not surprisingly, the GO analysis mainly identified GO terms related to lipid metabolism (Supplementary Fig. 2). Transcription factor analysis suggested Klf4 and SP1 as key drivers of the 

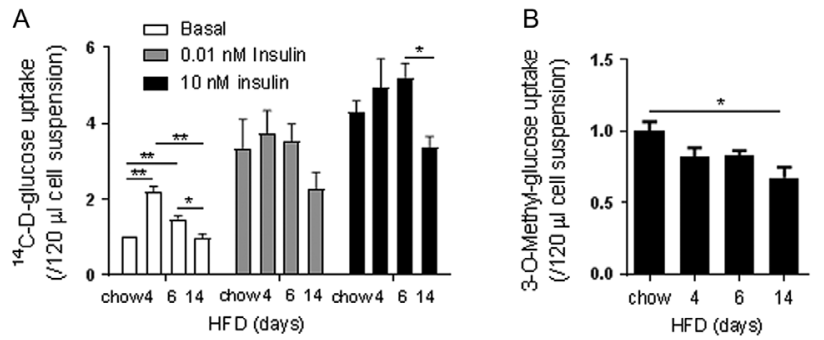

C
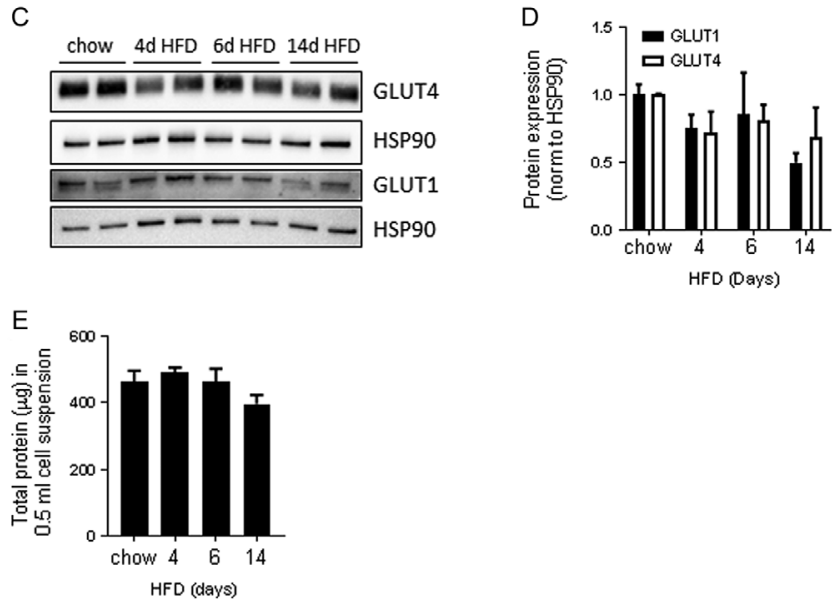

\section{Figure 4}

Intact glucose uptake despite cell expansion. Adipocytes were subjected to tracer glucose uptake assay, either non-stimulated (basal), insulin at $0.01 \mathrm{nM}$ or $10 \mathrm{nM}$ for $30 \mathrm{~min} . n=3$ independent experiments, each sample measured in triplicates and the mean of each triplicate was used for plotting the data. Data are normalized to lipid weight and expressed as uptake/cell suspension volume, relative to the non-stimulated uptake in the chow group (A). Adipocytes were subjected to 3-O-methyl glucose uptake assay to measure the cytosolic volume, $n=3$ independent experiments, each sample measured in triplicates, normalized to lipid weight and expressed as uptake/cell suspension volume, relative to chow group (B). Western blot analysis of adipose cell lysates for detection of GLUT1 and GLUT4, HSP90 used as loading control. 2 separate samples (biologic duplicate) were loaded/condition (C). Quantification of Western blot analysis of GLUT1 and GLUT4 $(n=2)(D)$. Total protein $(\mu \mathrm{g})$ content of an equal cell suspension volume $(0.5 \mathrm{~mL}), n=10$ individual samples/ feeding condition (E). Data in (A, B, D and E) presented as mean \pm S.E.M., ${ }^{*} P<0.05, * * P<0.01, * * * P<0.001$

transcriptional response at day 4 (Supplementary Fig. 1). Besides adipogenesis, Klf4 has previously been shown as a master regulator of autophagy in cardiomyocytes (Liao et al. 2015) and mouse fibroblasts (Liu et al. 2015). In addition, the most significantly upregulated gene at day 4

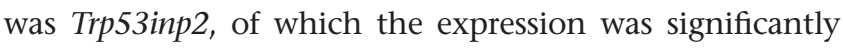
increased already after 2 days of HFD, which then remained elevated throughout the feeding protocol (Fig. 6D). Both Trp53inp2 and EHD2, the third top target (Fig. 6C) have recently been implicated in autophagy, by stimulating autophagosomal formation (Nowak et al. 2009) and in autophagic engulfment of lipid droplets (Li \& Schulze 2016).

\section{Increased autophagy in adipocytes from HFD-fed mice}

The prominent changes in autophagy-related gene expression and previous reports of altered autophagic flux in adipocytes from obese and diabetic subjects (Ost et al. 2010, Kovsan et al. 2011), prompted us to explore whether autophagy was altered in mature adipocytes already after 14 days of HFD. Mature adipocytes were isolated from mice fed either chow or HFD for 14 days, fixed and analyzed by confocal microscopy. Dispersed LC3-labeled structures were found throughout the adipocytes in cells isolated from both chow and HFDfed mice (representative images shown in Fig. 7A and B). Image analysis revealed a $\sim 2$.4-fold increase in density and an increased size (area) of the LC3-labeled puncta in adipocytes from HFD-fed mice (Fig. 7C and D). To measure the autophagic flux, cells were preincubated with chloroquine (CQ) to inhibit lysosomal degradation and rapamycin (Rap), which inhibits mTOR and thus stimulates autophagy (Ost et al. 2010). The pharmacological pre-treatment increased the density and size of the LC3-labeled puncta in both diet groups (Fig. 7C and D). The increase of LC3-labeled structures was most pronounced in cells from the chow-fed group ( 2.5-fold in chow versus $\sim 1.5$-fold in HFD), whereas the opposite was observed for the increase in size $(\sim 1.8$ fold in chow versus $\sim 3$-fold in the HFD group). By Western blot analysis, the LC3-II/I ratio was found to be markedly increased with HFD (Fig. 7E), illustrating increased lipidation of LC3-II, which is the LC3 isoform known to be localized to autophagosomal membranes (Kabeya et al. 2000). Rapamycin, alone or together with chloroquine, completely blocked the phosphorylation of pS6K1 Thr389, downstream of mTORC1, in both groups, confirming the efficacy of the pharmacologic treatment (Fig. 7E). Also, the level of p62, a protein which is degraded by autophagy (Bjorkoy et al. 2009), was lower after 14 days of HFD (Fig. 7F). Together, these observations suggest that autophagic activity is upregulated in adipocytes from short-term HFD-fed mice. In an attempt to assess whether or not autophagy in itself could affect glucose uptake capacity, adipocytes from chow-fed mice were pre-treated with rapamycin (Rap) for $4 \mathrm{~h}$ or left untreated and subjected to glucose uptake measurements (both non- and insulin stimulated). The pharmacological pre-treatment with rapamycin did not affect the glucose uptake capacity of the cells at any of the tested insulin concentrations (Fig. 7G). 
A

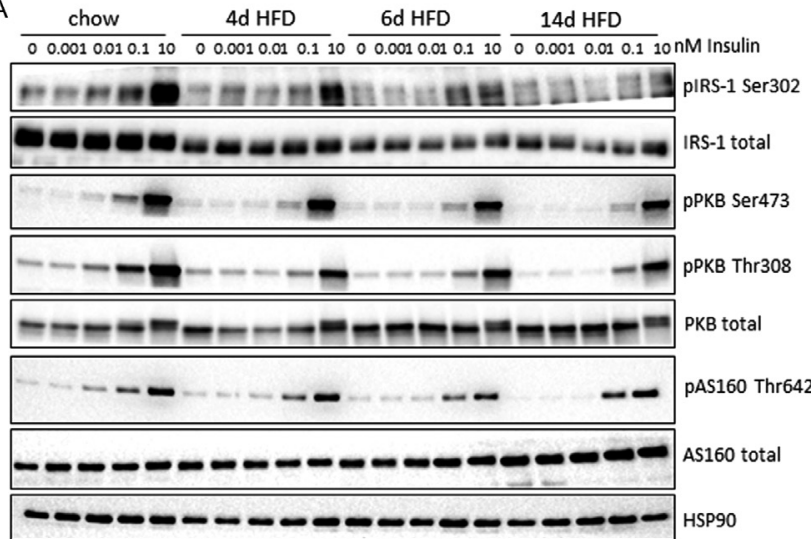

B
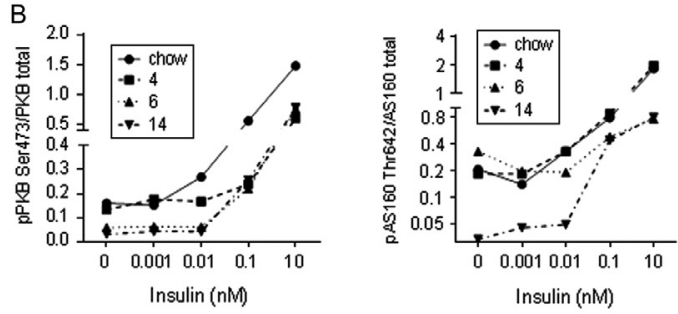

C

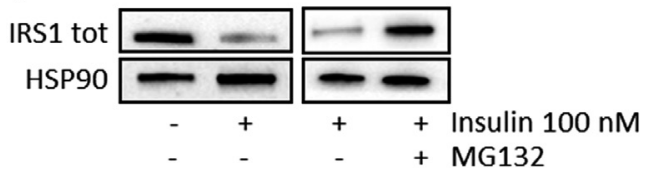

Figure 5

Progressively impaired submaximal insulin signaling. Adipocytes were either non-stimulated or stimulated with insulin $(0.001,0.01,0.1$ or $10 \mathrm{nM}$ ) for $30 \mathrm{~min}$ followed by Western blot analysis to detect total and phosphorylated protein levels of PKB and AS160, HSP90 used as loading control. Representative blots from $n=3$ independent experiments presented in (A). Western blot analysis of insulin-stimulated phosphorylation of PKB Ser473 and AS160 Thr642 in (A) was quantified and expressed as (phosphorylated protein/total protein), shown in (B). Representative images of Western blot analysis of cell lysates from mouse adipocytes treated for $18 \mathrm{~h}$ with MG132 $(10 \mu \mathrm{M})$ or vehicle (DMSO), with or without insulin (100 nM). HSP90 was used as loading control. ( $n=3$ independent experiments) (C).

\section{Discussion}

In a previous study, we developed a mathematical model of adipose tissue growth, suggesting that cellular hypertrophy occurs immediately after the onset of HFD feeding (Li et al. 2016). Here, we focused on characterizing adipocyte function during a similar short-term HFD feeding protocol to capture early cellular events preceding the onset of insulin resistance in adipocytes. Even though we detected reduced systemic insulin sensitivity already after two days of HFD, insulin-stimulated glucose uptake at the adipocyte level remained intact during the first six days of HFD. In parallel, we observed a rapid decrease in insulin- stimulated IRS-1 activation, as well as a gradual decrease in insulin-induced phosphorylation of $\mathrm{PKB}$, reflecting a decrease in insulin sensitivity, while the maximally stimulated response was preserved. The insulin-stimulated phosphorylation of AS160, a downstream target of PKB, was preserved to a larger extent, but was reduced after 14 days of HFD that coincided with a decrease in GLUT4 protein expression. At the same time, the adipocyte size in the large cell fraction had increased significantly. These findings could possibly explain the decreased insulinstimulated glucose uptake after 14 days of HFD and are in line with previous descriptions of large adipocytes as being less insulin responsive (Salans et al. 1968, Smith 1972) and having impaired GLUT4 translocation (Franck et al. 2007). The fact that we observed a decrease in PKB activation while insulin-stimulated glucose uptake remained intact, supports the description of spare insulin receptors (Kahn 1978), and the report of a switch-like behavior of PKB, where a smaller fraction of PKB phosphorylation was sufficient to stimulate a maximal downstream response (Tan et al. 2012). In cultured 3T3-L1 adipocytes, insulin resistance was associated with impaired AS160 phosphorylation and impaired GLUT4 translocation despite intact activation of upstream PKB (Tan et al. 2015). Our data suggest that phosphorylation of AS160, rather than IRS-1 and PKB, is a rate-limiting step for insulin-stimulated glucose uptake during early adipose tissue expansion. The observation that switching to a HFD induced a rapid decrease in IRS-1 protein in adipocytes was quite striking and could be due to insulin-mediated degradation, as previously reported (Buren et al. 2003). The fact that such a marked reduction in IRS-1 did not have a greater impact on the downstream targets PKB and AS160 could be due to an in-built over-capacity of signaling as described earlier and/or to compensatory mechanisms, potentially mediated by IRS-2 (Previs et al. 2000).

A rather unexpected finding was the increase in non-stimulated glucose uptake following 4 and 6 days of HFD. Since we could neither detect an increase in GLUT1 or GLUT4 at these time points, nor any radical changes in insulin signaling in the non-stimulated cells, we interpret this observation to reflect a higher uptake in smaller, newly recruited adipocytes. The presence of small adipocytes was supported by the cell size distribution data, which demonstrated a massive increase in the number of small cells at day 4 and by the transcription factor analysis, which implicated that Klf4, a regulator of adipogenesis, was prominent at that time. Also, one of the highly upregulated genes in adipose tissue following 4 days of HFD was TUSC5, recently reported to promote 
A

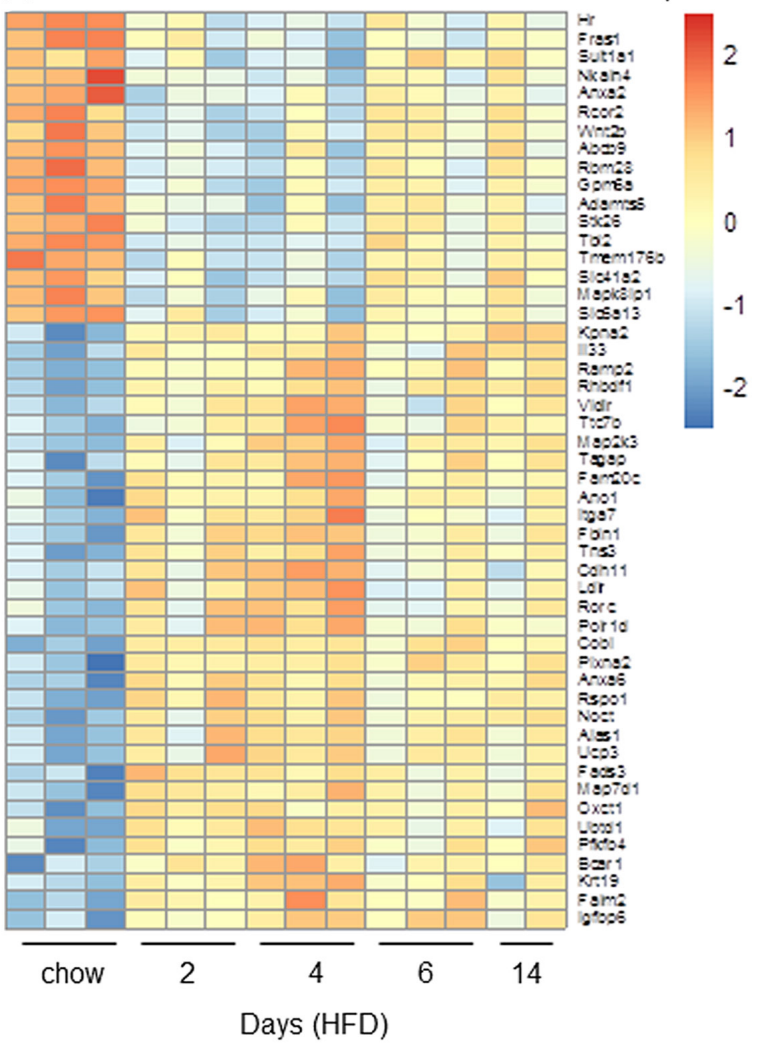

Expression

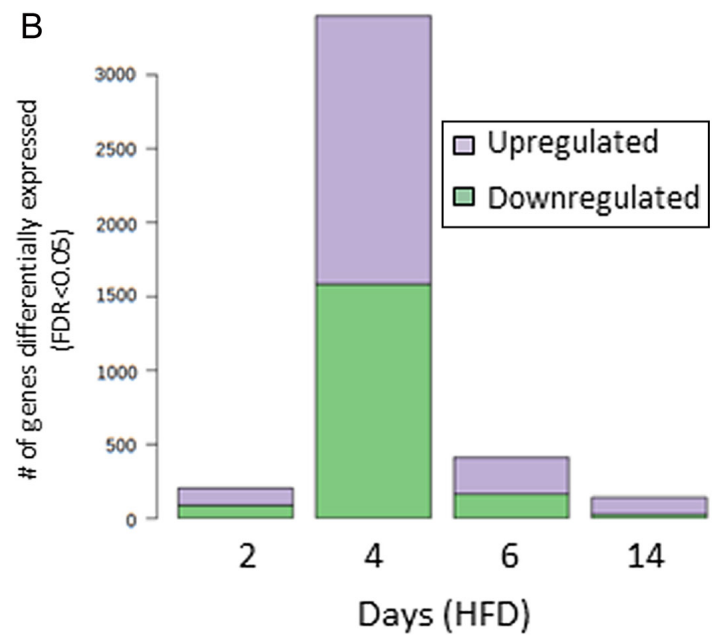

C

\begin{tabular}{|c|c|c|c|c|c|}
\hline Ranking & mai symbol & Description & $\begin{array}{l}\log F C \\
\text { (day } 4)\end{array}$ & $\begin{array}{l}\text { Average } \\
\text { expression }\end{array}$ & $\begin{array}{l}\text { Adjusted } \\
\text { P-value }\end{array}$ \\
\hline 1 & Trp53inp2 & $\begin{array}{l}\text { transformation related protein } 53 \\
\text { inducible nuclear protein } 2\end{array}$ & 1,37 & 10,0 & $8,1 \mathrm{E}-05$ \\
\hline 2 & Tusc5 & tumor suppressor candidate 5 & 1,02 & 9,2 & $8,1 \mathrm{E}-05$ \\
\hline 3 & Ehd2 & EH-domain containing 2 & 0,69 & 10,6 & $4,4 \mathrm{E}-04$ \\
\hline 4 & Slc6a13 & solute carrier family 6 & 0,86 & 7,0 & $5,5 \mathrm{E}-04$ \\
\hline 5 & D430019H16Rik & RIKEN CDNA D430019H16 gene & 1,46 & 6,5 & $6,8 \mathrm{E}-04$ \\
\hline 6 & Krt79 & keratin 79 & 1,76 & 3,5 & $8,0 \mathrm{E}-04$ \\
\hline 7 & Fads3 & fatty acid desaturase 3 & 0,69 & 9,2 & $8,7 \mathrm{E}-04$ \\
\hline 8 & Alas1 & aminolevulinic acid synthase 1 & 1,24 & 8,5 & $8,7 \mathrm{E}-04$ \\
\hline 9 & Rorc & RAR-related orphan receptor gamma & $-2,05$ & 1,8 & $9,6 \mathrm{E}-04$ \\
\hline 10 & Lgals1 & lectin, galactose binding, soluble 1 & 0,76 & 10,7 & $9,6 \mathrm{E}-04$ \\
\hline
\end{tabular}

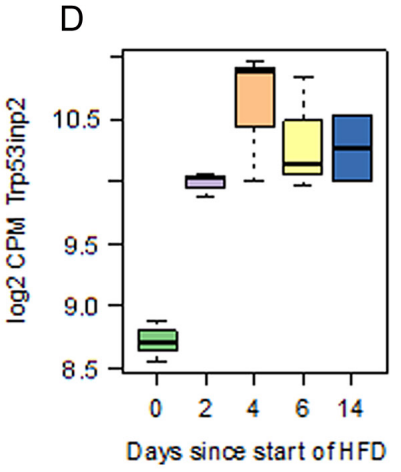

Figure 6

Profound transcriptional changes linked to autophagy following HFD feeding. Heatmap of the top fifty genes with highest moderated $F$-statistic at all time points generated using the R-package 'heatmap' Each gene (row) was $z$-score normalized (average expression across all samples $=0$ and s.D. $=1$ ) before visualization. The color indicates the expression of each gene with red being up-regulated and blue down-regulated (A). Number of differentially expressed genes at each time point (B). Differentially expressed genes were identified using the limma package and $P$-values were corrected using the FDR method. Each bar indicates the number of genes showing an FDR-corrected $P$-value $<0.05$ (B). Table of the ten most significant genes at day 4 compared to day 0 . ' $\log \mathrm{FC}^{\prime}$ denotes the log 2 ratio of day $4 /$ day 0 (positive value indicates up regulation at day 4). Average expression is in log 'counts per million' and is the average across all samples (C). Expression of the autophagy-related gene Trp53inp2 at day 0 (chow), 2, 4, 6 and 14 days of HFD (D).

insulin-stimulated glucose uptake in adipocytes by directing GLUT4 recycling (Beaton et al. 2015, Fazakerley et al. 2015). Possibly, increased expression of TUSC5 could account for the maintained or increased glucose uptake during the initial HFD feeding, thereby compensating for an impaired insulin signal transduction.
The measurements obtained in our assays reflect the total output from an entire population, with cells of varying sizes. Characterization of the cellular responses in the fractions containing small and large cells, respectively, would be of value, but are unfortunately technically challenging to perform in a robust fashion. 


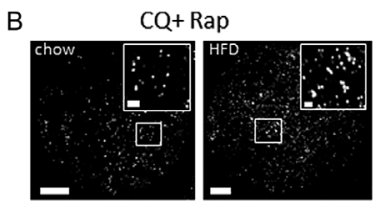

D
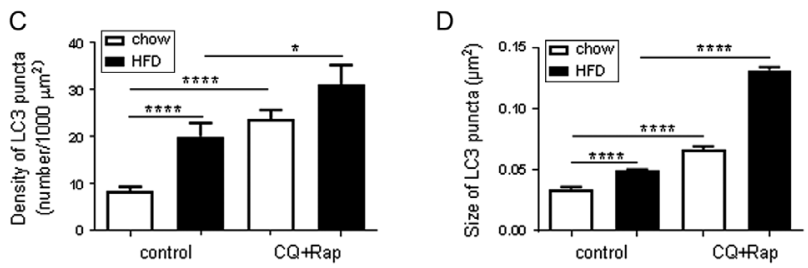

E

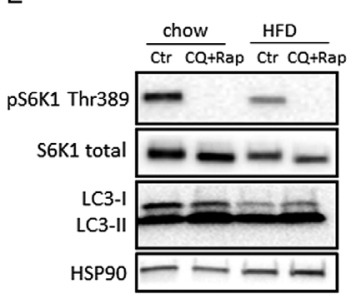

F

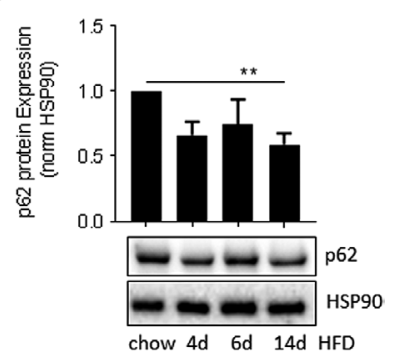

$\mathrm{G}$

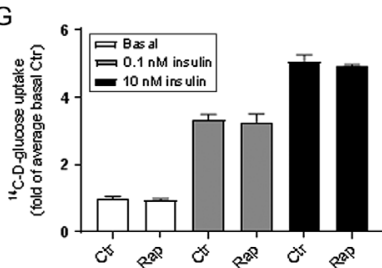

Figure 7

Increased autophagy in adipocytes from high fat diet fed mice. Representative images of adipocytes labeled with LC3 antibody, either non-treated (control) (A, left panel chow; right panel 14 days of HFD) or pre-incubated with chloroquine (CQ) and Rapamycin (Rap) for $4 \mathrm{~h}$ (B, left panel chow; right panel 14 days of HFD). LC3 labeling was analyzed and quantified, and data presented as density of LC3 puncta/1000 $\mu \mathrm{m}^{2}$ (C), and size of each LC3 puncta $\left(\mu \mathrm{m}^{2}\right)$ (D). In total, data from chow $n=114$ cells, HFD $n=96$ cells, chow Rap + CQ $n=116$ cells, HFD Rap + CQ $n=107$ cells, was collected for quantification in C and D. Representative images of Western blot analysis (S6K1 total and phosphorylated at Thr389, and LC3I/II) of cell lysates from cells either non-stimulated or pre-treated with either CQ, Rap or CQ and Rap in combination. HSP90 used as loading control. $n=2$ independent experiments (E). Quantification of p62 from Western blot analysis of cell lysates from mice fed HFD 4, 6 or 14 days (chow control). HSP90 used as loading control, $n=3$ independent experiments. Representative images of Western blot analysis below graph (F). Adipocytes from chow-fed mice were pre-treated with rapamycin (Rap, $500 \mathrm{nM}$ ) for $4 \mathrm{~h}$ or left untreated, and subjected to glucose uptake measurements (both non- and insulin stimulated) for $30 \mathrm{~min} . n=3$ independent experiments, each sample measured in triplicates and the mean of each triplicate was used for plotting the data (G). Data in ( $C$ and $\mathrm{D}, \mathrm{F}$ and $\mathrm{G}$ ) presented as mean \pm s.E.M., ${ }^{*} P<0.05, * * P<0.01, * * * * P<0.0001$.

Also, we were not able to determine the total cell number/cell suspension volume, but instead determined the cell size distribution in intact fat tissue from the same depot as the cells were isolated from. It is still reasonable to assume that the number of cells/volume

of cell suspension decreased with increasing days of HFD. Thus, the protein synthesis seemed to increase proportionally with cell expansion, even though there was a trend towards reduced protein content after 14 days, and certainly, a decrease in GLUT4 expression. Possibly, the decrease in cytosolic volume at the end of the feeding protocol (14 days of HFD) could contribute to a decreased protein synthesis and impaired metabolism. Still, even though there was an immediate downward shift in insulin signal transduction in adipocytes after switching to HFD feeding, the insulin-stimulated glucose uptake was preserved for an extended time.

Obesity-induced insulin resistance is commonly associated with infiltration of adipose tissue by inflammatory cells (Stienstra et al. 2011). However, we did not observe any pro-inflammatory response at the systemic level. Instead, our data support the previous notion that systemic insulin resistance following shortterm HFD feeding could be ascribed to ectopic fat storage in liver and/or muscle rather than a systemic inflammatory response (Lee et al. 2011).

Strikingly, the RNA-seq analysis revealed that the absolute majority of gene expression changes, either up or down, occurred at day 4, which we previously identified as the approximate time point of new adipocyte recruitment (Li et al. 2016). Future studies are required to explore whether adipose tissue alterations at this specific time-point could predict future adipocyte function, possibly revealing mechanisms explaining why some obese individuals develop insulin resistance, whereas other equally obese individuals remain healthy. We found several lines of evidence pin-pointing autophagy as one of the cellular processes that were quickly upregulated when switching to a HFD. Among the genes that displayed the most significant upregulation was Trp53inp2, which triggers autophagy by stimulating autophagosomal formation by interaction with LC3 and Beclin1 (Nowak et al. 2009). Several studies have highlighted the association of Trp53inp2 with autophagy (Nowak \& Iovanna 2009, Fromm-Dornieden et al. 2012, Mauvezin et al. 2012, Sala et al. 2014) and downregulation of the protein was reported to protect muscle tissue from autophagosomal degradation in type 2 diabetic subjects (Sala et al. 2014). Also, in line with our findings, the Trp53inp2 gene expression was upregulated in muscle and adipose tissue following 1 week of HFD (Fromm-Dornieden et al. 2012). In addition to the gene expression data, we demonstrate that the autophagic activity is upregulated in mature adipocytes from short-term HFD-fed mice. The decrease in p62 protein levels and the increase in 
LC3II after lysosomal inhibition also supports increased autophagy, as discussed by Barth and coworkers (Bjorkoy et al. 2009, Barth et al. 2010). Still, it's worth noting that the increase in LC3-positive puncta might reflect an impaired intracellular lipid clearance as previously reported (Singh et al. 2009). Even though species and cell type differences regarding autophagy have been reported, our data certainly fit previous observations in human adipocytes from type 2 diabetic patients, reporting overactive autophagy (Ost et al. 2010). In that study, increased autophagic flux was associated with impaired positive feedback to IRS-1 and diminished insulin signaling. Possibly, this could be a mechanism through which increased autophagy contributes to diminished insulin signaling, initially without affecting the glucose uptake in adipocytes. Indeed, when using rapamycin to induce autophagy in adipocytes from chow-fed mice we found no immediate effects on glucose uptake capacity after a 4-h treatment. Given the progressive deterioration in insulin signaling that we observed, it is however possible that autophagy, given enough time, might eventually lead or contribute to cellular insulin resistance and impaired glucose disposal. On the other hand, it is possible that the observed increase in autophagic activity instead serves a compensatory, protective function and that increased autophagy in adipocytes might help to initially maintain cellular function and systemic glucose homeostasis (Yang et al. 2010). In a recent report, mineralocorticoid receptor (MR) antagonists suppressed autophagy in white adipose tissue (Armani et al. 2014). We found no significant change in the MR expression, but the aldosterone signal pathway was upregulated and could thus provide a possible mechanistic link (Supplementary Fig. 3).

Together, we demonstrate that HFD feeding is associated with a rapidly impaired insulin-induced signal transduction at the level of IRS-1 and PKB in adipocytes, while insulin-stimulated glucose uptake remains intact. The increased autophagic activity during short-term HFD feeding suggests autophagy as one of the early cellular processes that could influence the metabolic function of adipocytes at an early stage of insulin resistance.

\section{Supplementary data}

This is linked to the online version of the paper at https://doi.org/10.1530/ JME-17-0195.

\section{Declaration of interest}

The authors declare that there is no conflict of interest that could be perceived as prejudicing the impartiality of the research reported.

\section{Funding}

This work was financially supported by the Swedish Research Council (2013-3542 to K.St.), Diabetes Foundation, The Crafoord Foundation, Albert Påhlsson Foundation and the Royal Physiographic Society in Lund.

\section{Acknowledgements}

The authors would like to thank Maria Lindahl for excellent technical support.

\section{References}

Al-Hasani H, Kunamneni RK, Dawson K, Hinck CS, Muller-Wieland D \& Cushman SW 2002 Roles of the N- and C-termini of GLUT4 in endocytosis. Journal of Cell Science 115 131-140.

Armani A, Cinti F, Marzolla V, Morgan J, Cranston GA, Antelmi A, Carpinelli G, Canese R, Pagotto U, Quarta C, et al. 2014 Mineralocorticoid receptor antagonism induces browning of white adipose tissue through impairment of autophagy and prevents adipocyte dysfunction in high-fat-diet-fed mice. FASEB Journal $\mathbf{2 8}$ 3745-3757. (https://doi.org/10.1096/fj.13-245415)

Barth S, Glick D \& Macleod KF 2010 Autophagy: assays and artifacts. Journal of Pathology 221 117-124. (https://doi.org/10.1002/ path.2694)

Beaton N, Rudigier C, Moest H, Muller S, Mrosek N, Roder E, Rudofsky G, Rulicke T, Ukropec J, Ukropcova B, et al. 2015 TUSC5 regulates insulin-mediated adipose tissue glucose uptake by modulation of GLUT4 recycling. Molecular Metabolism 4 795-810. (https://doi.org/10.1016/j.molmet.2015.08.003)

Bjorkoy G, Lamark T, Pankiv S, Overvatn A, Brech A \& Johansen T 2009 Monitoring autophagic degradation of p62/SQSTM1. Methods in Enzymology 452 181-197.

Buren J, Liu HX, Lauritz J \& Eriksson JW 2003 High glucose and insulin in combination cause insulin receptor substrate-1 and -2 depletion and protein kinase $\mathrm{B}$ desensitisation in primary cultured rat adipocytes: possible implications for insulin resistance in type 2 diabetes. European Journal of Endocrinology 148 157-167. (https://doi. org/10.1530/eje.0.1480157)

Danielsson A, Ost A, Lystedt E, Kjolhede P, Gustavsson J, Nystrom FH \& Stralfors P 2005 Insulin resistance in human adipocytes occurs downstream of IRS1 after surgical cell isolation but at the level of phosphorylation of IRS1 in type 2 diabetes. FEBS Journal $\mathbf{2 7 2}$ 141-151. (https://doi.org/10.1111/j.1432-1033.2004.04396.x)

Danielsson A, Fagerholm S, Ost A, Franck N, Kjolhede P, Nystrom FH \& Stralfors P 2009 Short-term overeating induces insulin resistance in fat cells in lean human subjects. Molecular Medicine 15 228-234.

Dobin A, Davis CA, Schlesinger F, Drenkow J, Zaleski C, Jha S, Batut P, Chaisson M \& Gingeras TR 2013 STAR: ultrafast universal RNA-seq aligner. Bioinformatics 29 15-21. (https://doi.org/10.1093/ bioinformatics/bts635)

Fazakerley DJ, Naghiloo S, Chaudhuri R, Koumanov F, Burchfield JG, Thomas KC, Krycer JR, Prior MJ, Parker BL, Murrow BA, et al. 2015 Proteomic analysis of GLUT4 storage vesicles reveals tumor suppressor candidate 5 (TUSC5) as a novel regulator of insulin action in adipocytes. Journal of Biological Chemistry 290 23528-23542. (https://doi.org/10.1074/jbc.M115.657361)

Folch J, Lees M \& Sloane Stanley GH 1957 A simple method for the isolation and purification of total lipides from animal tissues. Journal of Biological Chemistry 226 497-509.

Franck N, Stenkula KG, Ost A, Lindstrom T, Stralfors P \& Nystrom FH 2007 Insulin-induced GLUT4 translocation to the plasma membrane is blunted in large compared with small primary fat cells isolated 
from the same individual. Diabetologia 50 1716-1722. (https://doi. org/10.1007/s00125-007-0713-1)

Fromm-Dornieden C, Lytovchenko O, von der Heyde S, Behnke N, Hogl S, Berghoff J, Kopper F, Opitz L, Renne U, Hoeflich A, et al. 2012 Extrinsic and intrinsic regulation of DOR/TP53INP2 expression in mice: effects of dietary fat content, tissue type and sex in adipose and muscle tissues. Nutrition and Metabolism 9 86. (https://doi. org/10.1186/1743-7075-9-86)

Gliemann J, Rees WD \& Foley JA 1984 The fate of labelled glucose molecules in the rat adipocyte. Dependence on glucose concentration. Biochimica et Biophysica Acta 804 68-76. (https://doi. org/10.1016/0167-4889(84)90100-9)

Kabeya Y, Mizushima N, Ueno T, Yamamoto A, Kirisako T, Noda T, Kominami E, Ohsumi Y \& Yoshimori T 2000 LC3, a mammalian homologue of yeast Apg8p, is localized in autophagosome membranes after processing. EMBO Journal 19 5720-5728. (https:// doi.org/10.1093/emboj/19.21.5720)

Kahn CR 1978 Insulin resistance, insulin insensitivity, and insulin unresponsiveness: a necessary distinction. Metabolism: Clinical and Experimental 27 1893-1902. (https://doi.org/10.1016/S00260495(78)80007-9)

Kovsan J, Bluher M, Tarnovscki T, Kloting N, Kirshtein B, Madar L, Shai I, Golan R, Harman-Boehm I, Schon MR, et al. 2011 Altered autophagy in human adipose tissues in obesity. Journal of Clinical Endocrinology and Metabolism 96 E268-E277. (https://doi. org/10.1210/jc.2010-1681)

Krotkiewski M, Bjorntorp P, Sjostrom L \& Smith U 1983 Impact of obesity on metabolism in men and women. Importance of regional adipose tissue distribution. Journal of Clinical Investigation $\mathbf{7 2}$ 1150-1162. (https://doi.org/10.1172/JCI111040)

Lee YS, Li P, Huh JY, Hwang IJ, Lu M, Kim JI, Ham M, Talukdar S, Chen A, Lu WJ, et al. 2011 Inflammation is necessary for long-term but not short-term high-fat diet-induced insulin resistance. Diabetes 60 2474-2483. (https://doi.org/10.2337/db11-0194)

Li Z \& Schulze RJ 2016 A novel Rab10-EHBP1-EHD2 complex essential for the autophagic engulfment of lipid droplets. Science Advances 2 e1601470. (https://doi.org/10.1126/sciadv.1601470)

Li Y, Periwal V, Cushman SW \& Stenkula KG 2016 Adipose cell hypertrophy precedes the appearance of small adipocytes by 3 days in C57BL/6 mouse upon changing to a high fat diet. Adipocyte $\mathbf{5}$ 81-87. (https://doi.org/10.1080/21623945.2015.1128588)

Liao X, Zhang R, Lu Y, Prosdocimo DA, Sangwung P, Zhang L, Zhou G, Anand P, Lai L, Leone TC, et al. 2015 Kruppel-like factor 4 is critical for transcriptional control of cardiac mitochondrial homeostasis. Journal of Clinical Investigation 125 3461-3476. (https://doi. org/10.1172/JCI79964)

Lindahl M, Petrlova J, Dalla-Riva J, Wasserstrom S, Rippe C, DomingoEspin J, Kotowska D, Krupinska E, Berggreen C, Jones HA, et al. 2015 ApoA-I Milano stimulates lipolysis in adipose cells independently of cAMP/PKA activation. Journal of Lipid Research 56 2248-2259. (https://doi.org/10.1194/jlr.M054767)

Liu C, DeRoo EP, Stecyk C, Wolsey M, Szuchnicki M \& Hagos EG 2015 Impaired autophagy in mouse embryonic fibroblasts null for Kruppel-like factor 4 promotes DNA damage and increases apoptosis upon serum starvation. Molecular Cancer 14 101. (https://doi. org/10.1186/s12943-015-0373-6)

Mauvezin C, Sancho A, Ivanova S, Palacin M \& Zorzano A 2012 DOR undergoes nucleo-cytoplasmic shuttling, which involves passage through the nucleolus. FEBS Letters 586 3179-3186. (https://doi. org/10.1016/j.febslet.2012.06.032)

McLaughlin T, Sherman A, Tsao P, Gonzalez O, Yee G, Lamendola C, Reaven GM \& Cushman SW 2007 Enhanced proportion of small adipose cells in insulin-resistant vs insulin-sensitive obese individuals implicates impaired adipogenesis. Diabetologia 50 1707-1715. (https://doi.org/10.1007/s00125-007-0708-y)
Nowak J \& Iovanna JL 2009 TP53INP2 is the new guest at the table of self-eating. Autophagy 5 383-384. (https://doi.org/10.4161/ auto.5.3.7698)

Nowak J, Archange C, Tardivel-Lacombe J, Pontarotti P, Pebusque MJ, Vaccaro MI, Velasco G, Dagorn JC \& Iovanna JL 2009 The TP53INP2 protein is required for autophagy in mammalian cells. Molecular Biology of the Cell 20 870-881. (https://doi.org/10.1091/mbc.E08-07-0671)

Nunez CE, Rodrigues VS, Gomes FS, Moura RF, Victorio SC, Bombassaro B, Chaim EA, Pareja JC, Geloneze B, Velloso LA, et al. 2013 Defective regulation of adipose tissue autophagy in obesity. International Journal of Obesity 37 1473-1480. (https://doi. org/10.1038/ijo.2013.27)

Ost A, Svensson K, Ruishalme I, Brannmark C, Franck N, Krook H, Sandstrom P, Kjolhede P \& Stralfors P 2010 Attenuated mTOR signaling and enhanced autophagy in adipocytes from obese patients with type 2 diabetes. Molecular Medicine 16 235-246. (https://doi.org/10.1007/s00894-009-0539-5)

Pacini G, Omar B \& Ahren B 2013 Methods and models for metabolic assessment in mice. Journal of Diabetes Research 2013986906. (https://doi.org/10.1155/2013/986906)

Previs SF, Withers DJ, Ren JM, White MF \& Shulman GI 2000 Contrasting effects of IRS-1 versus IRS-2 gene disruption on carbohydrate and lipid metabolism in vivo. Journal of Biological Chemistry 275 38990-38994. (https://doi.org/10.1074/jbc. M006490200)

Rodbell M 1964 Metabolism of isolated fat cells. I. Effects of hormones on glucose metabolism and lipolysis. Journal of Biological Chemistry 239 375-380.

Rotter V, Nagaev I \& Smith U 2003 Interleukin-6 (IL-6) induces insulin resistance in 3T3-L1 adipocytes and is, like IL-8 and tumor necrosis factor-alpha, overexpressed in human fat cells from insulin-resistant subjects. Journal of Biological Chemistry 278 45777-45784. (https:// doi.org/10.1074/jbc.M301977200)

Sala D, Ivanova S, Plana N, Ribas V, Duran J, Bach D, Turkseven S, Laville M, Vidal H, Karczewska-Kupczewska M, et al. 2014 Autophagy-regulating TP53INP2 mediates muscle wasting and is repressed in diabetes. Journal of Clinical Investigation 124 1914-1927. (https://doi.org/10.1172/JCI72327)

Salans LB \& Dougherty JW 1971 The effect of insulin upon glucose metabolism by adipose cells of different size. Influence of cell lipid and protein content, age, and nutritional state. Journal of Clinical Investigation 50 1399-1410. (https://doi.org/10.1172/JCI106623)

Salans LB, Knittle JL \& Hirsch J 1968 The role of adipose cell size and adipose tissue insulin sensitivity in the carbohydrate intolerance of human obesity. Journal of Clinical Investigation 47 153-165. (https:// doi.org/10.1172/JCI105705)

Salans LB, Cushman SW \& Weismann RE 1973 Studies of human adipose tissue. Adipose cell size and number in nonobese and obese patients. Journal of Clinical Investigation 52 929-941. (https://doi. org/10.1172/JCI107258)

Shao J, Yamashita H, Qiao L \& Friedman JE 2000 Decreased Akt kinase activity and insulin resistance in C57BL/KsJ-Leprdb/db mice. Journal of Endocrinology 167 107-115. (https://doi.org/10.1677/ joe.0.1670107)

Singh R, Kaushik S, Wang Y, Xiang Y, Novak I, Komatsu M, Tanaka K, Cuervo AM \& Czaja MJ 2009 Autophagy regulates lipid metabolism. Nature 458 1131-1135. (https://doi.org/10.1038/nature07976)

Smith U 1972 Studies of human adipose tissue in culture. I. Incorporation of glucose and release of glycerol. Anatomical Record 172 597-602. (https://doi.org/10.1002/ar.1091720401)

Soussi H, Reggio S, Alili R, Prado C, Mutel S, Pini M, Rouault C, Clement K \& Dugail I 2015 DAPK2 downregulation associates with attenuated adipocyte autophagic clearance in human obesity. Diabetes 64 3452-3463. (https://doi.org/10.2337/db141933)
(C) 2018 Society for Endocrinology Published by Bioscientifica Ltd. Printed in Great Britain 
Stienstra R, van Diepen JA, Tack CJ, Zaki MH, van de Veerdonk FL, Perera D, Neale GA, Hooiveld GJ, Hijmans A, Vroegrijk I, et al. 2011 Inflammasome is a central player in the induction of obesity and insulin resistance. PNAS 108 15324-15329. (https://doi.org/10.1073/ pnas.1100255108)

Tan SX, Ng Y, Meoli CC, Kumar A, Khoo PS, Fazakerley DJ, Junutula JR, Vali S, James DE \& Stockli J 2012 Amplification and demultiplexing in insulin-regulated Akt protein kinase pathway in adipocytes. Journal of Biological Chemistry 287 6128-6138. (https://doi. org/10.1074/jbc.M111.318238)

Tan SX, Fisher-Wellman KH, Fazakerley DJ, Ng Y, Pant H, Li J, Meoli CC, Coster AC, Stockli J \& James DE 2015 Selective insulin resistance in adipocytes. Journal of Biological Chemistry 290 11337-11348. (https:// doi.org/10.1074/jbc.M114.623686)

Weyer C, Foley JE, Bogardus C, Tataranni PA \& Pratley RE 2000 Enlarged subcutaneous abdominal adipocyte size, but not obesity itself, predicts type II diabetes independent of insulin resistance.
Diabetologia 43 1498-1506. (https://doi.org/10.1007/ s001250051560)

Whitesell RR \& Gliemann J 1979 Kinetic parameters of transport of 3-O-methylglucose and glucose in adipocytes. Journal of Biological Chemistry 254 5276-5283.

Winzell MS \& Ahren B 2004 The high-fat diet-fed mouse: a model for studying mechanisms and treatment of impaired glucose tolerance and type 2 diabetes. Diabetes 53 (Supplement 3) S215-S219. (https:// doi.org/10.2337/diabetes.53.suppl_3.S215)

Yang L, Li P, Fu SN, Calay ES \& Hotamisligil GS 2010 Defective hepatic autophagy in obesity promotes ER stress and causes insulin resistance. Cell Metabolism 11 467-478. (https://doi.org/10.1016/j. cmet.2010.04.005)

Yang J, Eliasson B, Smith U, Cushman SW \& Sherman AS 2012 The size of large adipose cells is a predictor of insulin resistance in firstdegree relatives of type 2 diabetic patients. Obesity 20 932-938. (https://doi.org/10.1038/oby.2011.371)

Received in final form 8 January 2018

Accepted 16 January 2018

Accepted Preprint published online 16 January 2018 